\title{
Coffee consumption and mortality in three Eastern European countries: results from the HAPIEE (Health, Alcohol and Psychosocial factors In Eastern Europe) study
}

\author{
Giuseppe Grosso 1,2,*, Urszula Stepaniak ${ }^{2}$, Agnieszka Micek², Denes Stefler ${ }^{3}$, \\ Martin Bobak ${ }^{3}$ and Andrzej Pajak ${ }^{2}$ \\ ${ }^{1}$ Integrated Cancer Registry of Catania-Messina-Siracusa-Enna, Azienda Ospedaliero Universitaria Policlinico \\ Vittorio Emanuele, Via S. Sofia 85, 95123 Catania, Italy: ${ }^{2}$ Department of Epidemiology and Population Studies, \\ Jagiellonian University Medical College, Krakow, Poland: ${ }^{3}$ Department of Epidemiology and Public Health, \\ University College London, London, UK
}

Submitted 24 March 2016: Final revision received 31 May 2016: Accepted 2 June 2016: First published online 14 July 2016

\begin{abstract}
Objective: To test the association between coffee consumption and risk of all-cause, CVD and cancer death in a European cohort.

Design: Prospective cohort study. Cox proportional hazards models with adjustment for potential confounders to estimate multivariable hazard ratios (HR) and 95\% CI were used.

Setting: Czech Republic, Russia and Poland.

Subjects: A total of 28561 individuals followed for $6 \cdot 1$ years.

Results: A total of 2121 deaths (43.1\% CVD and 35.7\% cancer mortality) occurred during the follow-up. Consumption of 3-4 cups coffee/d was associated with lower mortality risk in men $(\mathrm{HR}=0.83 ; 95 \% \mathrm{CI} 0.71,0.99)$ and women $(\mathrm{HR}=0.63 ; 95 \% \mathrm{CI}$ $0.47,0.84)$, while further intake showed non-significant reduced risk estimates $(\mathrm{HR}=0.71 ; 95 \% \mathrm{CI} 0.49,1.04$ and $\mathrm{HR}=0.51 ; 95 \% \mathrm{CI} 0.24,1.10$ in men and women, respectively). Decreased risk of CVD mortality was also found in men (HR $=0 \cdot 71$; $95 \%$ CI $0 \cdot 54,0 \cdot 93$ ) for consumption of 3-4 cups coffee/d. Stratified analysis revealed that consumption of a similar amount of coffee was associated with decreased risk of all-cause ( $\mathrm{HR}=0.61 ; 95 \% \mathrm{CI} 0.43,0.87)$ and cancer mortality ( $\mathrm{HR}=0.59 ; 95 \% \mathrm{CI}$ $0.35,0.99)$ in non-smoking women and decreased risk of all-cause mortality for $>4$ cups coffee/d in men with no/moderate alcohol intake.

Conclusions: Coffee consumption was associated with decreased risk of mortality. The protective effect was even stronger when stratification by smoking status and alcohol intake was performed.
\end{abstract}

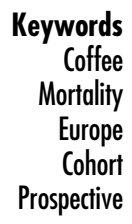

Coffee is among the most consumed non-alcoholic beverages worldwide ${ }^{(1)}$. Besides the gastronomic aspects, coffee has been suggested to affect human health and disease ${ }^{(2)}$. Although in the past it has been considered unhealthful due to its association with some negative outcomes following acute ingestion (i.e. rise in blood pressure and plasma lipids, inhibition of insulin activity), adverse health outcomes of long-term coffee consumption have not been found. Indeed, several epidemiological studies have explored the relationship between coffee intake and cardiovascular risk factors ${ }^{(3,4)}, \mathrm{CVD}^{(5)}$ and certain types of cancer $^{(6)}$, suggesting that a protective effect may exist. From an epidemiological point of view, long-term coffee drinking exerts different effects from acute intake typically examined under experimental conditions. Long-term experimental studies have demonstrated that habitual coffee drinkers develop tolerance to the acute effects of caffeine and may experience the effects of other coffee compounds that are related with its beneficial role on human health ${ }^{(7)}$. Coffee is a complex mixture of several biochemically active components, including caffeine, phenolic compounds and minerals (magnesium and potassium). Many of these compounds may act as antioxidants, thus preventing the state of subclinical inflammation involved in the pathogenesis of diseases such as cancer and $\mathrm{CVD}^{(8)}$. Among them, chlorogenic acids are responsible for ameliorating hypertension, glucose and lipid metabolism, whereas diterpenes demonstrate anti-carcinogenic properties $^{(9)}$.

Recently published prospective studies evaluating the association between coffee consumption and mortality in 
large European cohorts have been mostly conducted in Northern ${ }^{(10-12)}$ and Western European countries ${ }^{(13)}$. Findings obtained are puzzling and inconsistent, suggesting that significant differences between countries and methods of coffee consumption may be relevant for the outcome explored. We recently reported an inverse association between coffee consumption and metabolic impairment and decreased risk of developing hypertension in Polish individuals free of diseases at baseline ${ }^{(14,15)}$. However, whether these associations may be translated into a decreased risk of mortality following a prospective evaluation is unknown. To date, no further data from Eastern European countries are available. The aim of the present study was to test the association between coffee consumption and risk of all-cause, CVD and cancer mortality in the HAPIEE (Health, Alcohol and Psychosocial factors In Eastern Europe) study, a large-scale cohort study including adult individuals from the Czech Republic, Russia and Poland.

\section{Methods}

\section{Study population}

The HAPIEE study is a multicentre prospective cohort study investigating the role of biological, dietary, lifestyle and environmental factors in cardiovascular and other chronic diseases ${ }^{(16)}$. The criteria for sample selection and the methods are reported in detail elsewhere ${ }^{(16)}$. Briefly, a random sample of 28945 individuals (aged 4569 years) was recruited at the baseline survey conducted in 2002-2005 (response ratio of 59\%) in Novosibirsk (Russia), Krakow (Poland) and six Czech urban centres. The participants, after providing written informed consent, filled in a structured questionnaire and were invited to a clinical examination. Participants who left more than $50 \%$ of food items blank in the FFQ or reported implausible energy (energy intake $<2092 />16736 \mathrm{~kJ} / \mathrm{d}$ $(<500 />4000 \mathrm{kcal} / \mathrm{d})$ for females and $<3347 />20920 \mathrm{~kJ} / \mathrm{d}$ ( $<800 />5000 \mathrm{kcal} / \mathrm{d}$ ) for males, $n$ 384) were excluded from the analyses. A total of 28561 individuals were included for the final analysis. The study was conducted according to the guidelines laid down in the Declaration of Helsinki and all procedures involving human subjects were approved by the University College London Hospital research ethics committee and by ethical committees in each participating country. Written informed consent was obtained from all subjects.

\section{Demographic, lifestyle and clinical measurements} Sociodemographic and lifestyle characteristics included age, gender, educational and occupational level, smoking and alcohol drinking habits. Physical activity included energy expenditure both at work and leisure time. The overall amount of energy expenditure was calculated and categorized as low, moderate and high activity level.
Individuals were categorized according their smoking status as non-smoker and current smoker. Alcohol consumption was categorized as none or low $(\leq 12 \mathrm{~g} / \mathrm{d})$ and moderate to high alcohol drinker $(>12 \mathrm{~g} / \mathrm{d})$. The physical examination included measurement of height, weight and blood pressure using standard procedures ${ }^{(16)}$. BMI was calculated according the formula [weight $(\mathrm{kg})$ ]/ [height $(\mathrm{m})]^{2}$. Blood pressure was measured three times at the end of the physical examination and the final value was the mean among the three measurements. Participants were categorized as having hypertension at baseline if they had a systolic and/or a diastolic blood pressure measurement higher than 139 and/or $89 \mathrm{mmHg}$, respectively, or had taken hypertensive medication within the last 2 weeks. Participants were categorized as having diabetes at baseline if they had fasting plasma glucose $>120 \mathrm{mg} / \mathrm{dl}$, previously diagnosed type 2 diabetes or were receiving treatment for diabetes. Participants were categorized as having hypercholesterolaemia if they were previously diagnosed with or using a specific treatment for this lipid abnormality. Presence of CVD or cancer relied on previous diagnosis. Participants were finally asked whether they also had family history of CVD or cancer.

\section{Dietary assessment}

Dietary data were collected by using an FFQ based on the tool developed by Willett et al. ${ }^{(17)}$ and subsequently adapted in the Whitehall II Study ${ }^{(18)}$. The Czech, Russian and Polish FFQ consisted of 136, 147 and 148 food and drink items, respectively (the different numbers of questions are due to country-specific dishes). An instruction manual, which included photographs to facilitate the estimation of portion sizes, was used. Participants were asked how often, on average, they had consumed that amount of the item during the last 3 months, with nine responses ranging from 'never or less than once per month' to 'six or more times per day'. Moreover, participants were asked to include additional foods and frequency of consumption by manual entry.

The average beverage consumption was calculated (in millilitres) by following the standard portion sizes used in the study and then converted into $24 \mathrm{~h}$ intake. We categorized daily coffee consumption according to standard cups of coffee $(150 \mathrm{ml})$ as: (i) $<1$ cup/d; (ii) 1-2 cups/d; (iii) 3-4 cups/d; and (iv) $>4 \mathrm{cups} / \mathrm{d}$.

\section{Mortality}

The mortality status of participants was obtained through linkage with mortality registers of their respective country. Specifically, mortality data in Russia were obtained from the death register developed by the Institute of Internal Medicine, based on data from medical death certificates, the Novosibirsk office of the State Statistical Bureau and the population registration bureau. In Poland, data from the Local Register of Residents of the City of Krakow and 
Central Statistical Office were used. In the Czech Republic, data from the National Death Register were used. The follow-up period was until December 2011 for the Czech Republic or December 2010 for Russia and Poland. Participants who were lost to follow-up were censored at their last date of contact ( $n$ 527). The underlying cause of death was assigned according to the International Classification of Diseases, 10th Revision (ICD-10). The primary end point in the current analysis was death from any cause. We also conducted analyses according to CVD (ICD-10 codes I00-I99, CHD codes I20-I25, stroke codes I60-I69) and cancer mortality (codes C00-D48).

\section{Statistical analysis}

The distribution of background characteristics was compared between different coffee consumption groups using ANOVA or the Mantel-Haenszel test. We calculated person-years of follow-up for each participant from the date of the baseline questionnaire to the date of death from any cause, loss to follow-up or end of the follow-up period, whichever came first. Hazard ratios (HR) and 95\% CI for the association between coffee consumption and all end points, using the lowest category as the reference, were calculated by Cox proportional hazards models. No interactions between countries and categories of coffee consumption were detected, so we calculated the results of the Cox regression in the pooled sample and checked possible differences in an additional analysis by country cohorts. A crude model was adjusted for age (continuous) and gender. A multivariate model was built adjusting for country (Poland, Czech Republic, Russia), educational level (low, medium, high), physical activity (low, medium, high), smoking status (yes/no), history of CVD or cancer (yes/no), health status (presence of hypertension, diabetes, use of cholesterollowering medications), family history of CVD (yes/no), family history of cancer (yes/no), menopausal status in women (yes/no), alcohol intake $>12 \mathrm{~g} / \mathrm{d}$ (yes/no), total energy intake (continuous), SFA intake (continuous), PUFA intake (continuous), $n-3$ PUFA intake (continuous) and use of vitamin supplements (yes/no). The analysis was performed also for CVD and cancer mortality and stratified by gender and smoking status. To evaluate reverse causation, the risk of mortality excluding deaths occurring in the first 2 years from baseline, in individuals with a past history of CVD or cancer, and after sequential exclusion of individuals with CVD risk factors (hypertension, diabetes, use of cholesterol-lowering medications), was also estimated. All reported $P$ values were based on two-sided tests and compared with a significance level of $5 \%$. Analyses were performed using the statistical software package SPSS Statistics Version 17.0.

\section{Results}

A total of 2121 deaths (1435 men and 686 women) occurred during a mean follow-up of $6 \cdot 1$ years. Of these deaths, 913 (43.1\%) were due to CVD and 755 (35.7\%) were due to cancer mortality. Distribution of background characteristics by categories of coffee consumption did not differ significantly between men and women (Table 1). No specific trends were identified, with the exception of the distribution of current smokers (increasing with increased category of coffee consumption) and prevalence of hypertension and CVD (decreasing with increased category of coffee consumption). High alcohol intake was more frequent among men than women (about 18-20\% $v$. $1-4 \%$, respectively) and associated with higher coffee consumption, whereas prevalence of hypercholesterolaemia and family history of CVD and cancer were higher in women than in men.

Frequency and risk of all-cause, CVD and cancer mortality based on coffee consumption are shown in Table 2. Risk of all-cause mortality decreased with increasing coffee consumption in both men and women, both in age-adjusted and multivariate-adjusted models, with no significant difference in the distribution of deaths across categories of exposure between genders $(P=0 \cdot 094$; Table 2). A significant risk reduction of $17 \%(\mathrm{HR}=0 \cdot 83$; $95 \%$ CI $0.71,0.99)$ in men and $37 \%(\mathrm{HR}=0.63$; $95 \% \mathrm{CI}$ $0 \cdot 47,0.84$ ) in women was found for consumption of 3-4 cups/d, whereas further intake led to a non-significant decrease in risk. The risk estimate of all-cause mortality by category of coffee consumption partially reflected the risk of mortality by CVD in both women and men, whereas non-significant decreased risk in women and nonsignificant increased in men for risk cancer mortality was found. The stratified analysis by country revealed consistent non-significant decreasing mortality risk estimates with increasing coffee consumption across all cohorts and significant decreased risk up to $4 \mathrm{cups} / \mathrm{d}$ or less (see online supplementary material, Supplemental Table 1). Moreover, the analysis revealed that the Russian cohort was the only one showing an increased nonsignificant risk of death by cancer, whereas the others showed a decreased, non-significant risk.

A number of analyses to evaluate possible reverse causation are reported in the online supplementary material, Supplemental Table 2. Analysis performed by excluding deaths occurring within the first 2 years from baseline of follow-up showed no significant alteration of the results among both men and women. No substantially different risks were estimated when excluding individuals with a past history of CVD or cancer. Sequential exclusion also of individuals with any CVD risk factors weakened CVD mortality risk in women due to the limited cases in the highest categories of exposure, whereas it led to a non-significant decreased risk of death from cancer in men consumers of 3-4 cups coffee/d.

Results of stratified analyses by smoking status and alcohol intake are presented in Tables 3 and 4 , respectively. Distribution of deaths across categories of exposure between smokers and non-smokers was 


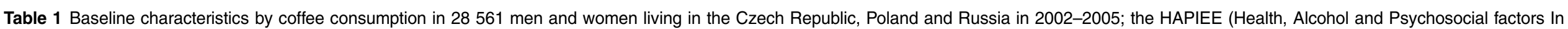
Eastern Europe) study

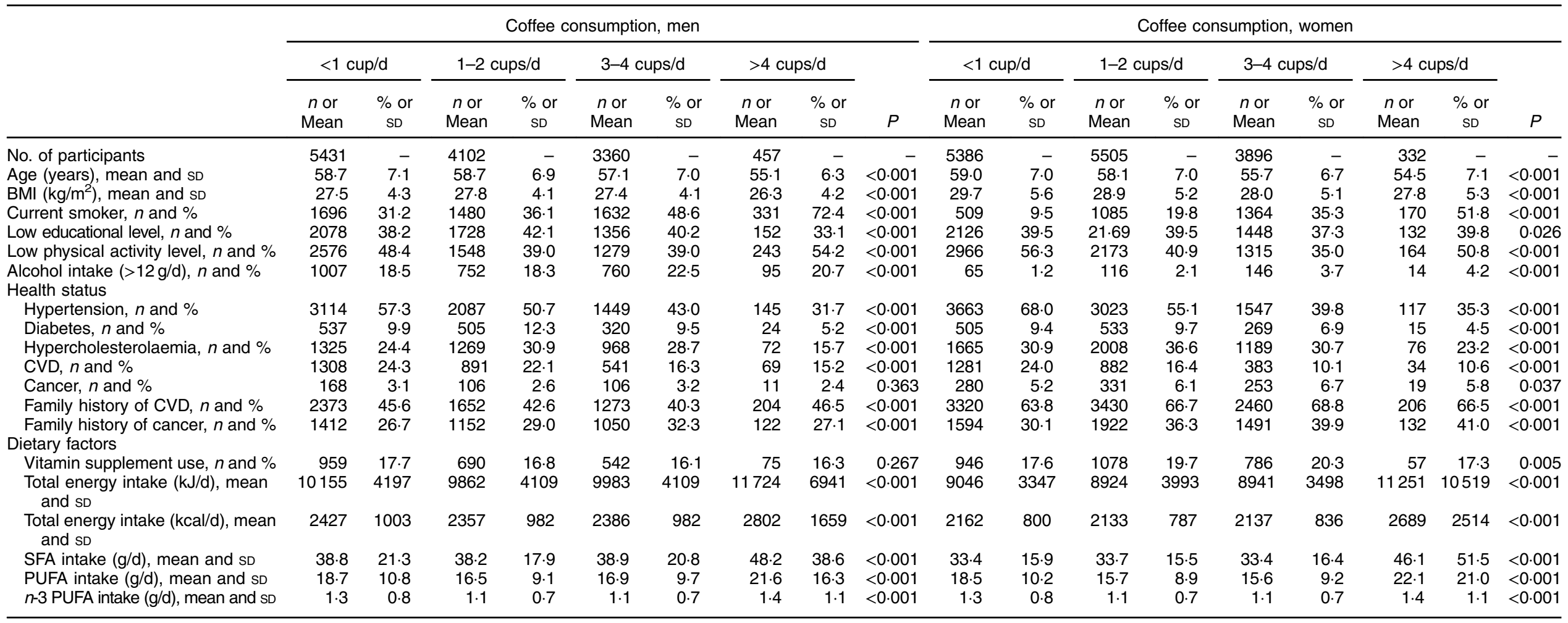


significantly different, with more numerous mortality cases among the categories of higher coffee intake in smokers than in non-smokers $(P<0 \cdot 001)$. Nevertheless, consumption of 3-4 cups coffee/d was associated with significantly decreased risk of CVD mortality in smoker men (HR= $0.66 ; 95 \% \mathrm{CI} 0.47,0.93)$ and decreased risk of all-cause $(\mathrm{HR}=0.61 ; 95 \%$ CI $0.43,0.87)$ and cancer mortality $(\mathrm{HR}=0.59 ; 95 \% \mathrm{CI} 0.35,0.99)$ in non-smoker women (Table 3). Results were mainly confirmed after stratification by alcohol consumption, with no significant difference between groups $(P=0 \cdot 068$; Table 4$)$. However, among men with alcohol intake of $\leq 12 \mathrm{~g} / \mathrm{d}$, consumption of $>4$ cups coffee/d was associated with a decreased risk of all-cause mortality ( $\mathrm{HR}=0.61 ; 95 \%$ CI $0.39,0.94)$.

\section{Discussion}

In the present study we found that daily coffee consumption was significantly correlated with decreased risk of all-cause and CVD death, but the effect was not observed when drinking more than 4 cups/d. Interestingly, we also found that after stratifying the sample by smoking status, coffee consumption was inversely associated with risk of death by cancer in women drinking 3-4 cups coffee/d. Lack of decreased risk of death for higher intakes of coffee has been recently reported, suggesting that the association of coffee drinking with mortality risk may not be linear ${ }^{(19)}$. These findings are in line with a recent dose-response metaanalysis ${ }^{(20)}$. It has been hypothesized that intake of high quantities of coffee may have an acute effect at the vascular level by triggering coronary events and, consequently, increasing the risk of myocardial infarction in specific patient groups, such as the elderly and those at high CVD risk ${ }^{(21,22)}$. This may depend on the biological effect of caffeine intake that causes an acute increase in arterial wave reflection, which in turn increases the pulsatile load of the heart. Furthermore, it has been reported that excessive intakes may also aggravate cardiac arrhythmias and raise plasma homocysteine ${ }^{(23)}$. However, other studies have reported that habitual coffee consumption is not directly associated with myocardial infarction in the general population and that the effects of caffeine in habitual coffee drinkers are attenuated or overall absent due to the development of tolerance $e^{(7,24-27)}$. The contrasting hypotheses on the effects of caffeine on cardiovascular events may reflect the modality of coffee consumption. Indeed, a habitual, rather than occasional intake of coffee may lead to desensitization to the acute effects of caffeine but excessive intake (i.e. more than 4 cups/d) could exacerbate its harmful effects.

The analysis of cancer mortality according coffee consumption revealed non-significant associations towards both increased and decreased risk, but after stratifying the analysis by smoking status, consumption of 3-4 cups coffee/d was associated with a decreased risk of death from cancer of about $40 \%$ compared with non-drinkers. Some 
Table 3 Stratified analysis by smoking status of age- and multivariate-adjusted hazard ratios (HR) and $95 \%$ confidence intervals for all-cause, CVD and cancer mortality by coffee consumption in men and women living in the Czech Republic, Poland, and Russia over a period of 6 years; the HAPIEE (Health, Alcohol and Psychosocial factors In Eastern Europe) study

\begin{tabular}{|c|c|c|c|c|c|c|c|c|c|c|c|c|c|c|}
\hline & \multicolumn{7}{|c|}{ Coffee consumption, men } & \multicolumn{7}{|c|}{ Coffee consumption, women } \\
\hline & \multirow{2}{*}{$\frac{<1 \text { cup/d }}{\text { Ref. }}$} & \multicolumn{2}{|c|}{$1-2$ cups/d } & \multicolumn{2}{|c|}{$3-4$ cups/d } & \multicolumn{2}{|c|}{$>4 \mathrm{cups} / \mathrm{d}$} & \multirow{2}{*}{$\frac{<1 \text { cup/d }}{\text { Ref. }}$} & \multicolumn{2}{|c|}{$1-2$ cups $/ \mathrm{d}$} & \multicolumn{2}{|c|}{$3-4$ cups $/ \mathrm{d}$} & \multicolumn{2}{|c|}{$>4$ cups $/ \mathrm{d}$} \\
\hline & & $\mathrm{HR}$ & $95 \% \mathrm{Cl}$ & $\mathrm{HR}$ & $95 \% \mathrm{Cl}$ & HR & $95 \% \mathrm{Cl}$ & & HR & $95 \% \mathrm{Cl}$ & $\mathrm{HR}$ & $95 \% \mathrm{Cl}$ & $\mathrm{HR}$ & $95 \% \mathrm{Cl}$ \\
\hline \multicolumn{15}{|l|}{ Smokers } \\
\hline \multicolumn{15}{|l|}{ Death from all causes } \\
\hline Person-years & 9604 & 8348 & - & 9782 & - & 2003 & - & 2861 & 6245 & - & 8689 & - & 1146 & - \\
\hline No. of deaths & 293 & 209 & - & 178 & - & 33 & - & 48 & 63 & - & 58 & - & 8 & - \\
\hline Multivariate-adjusted ${ }^{*}$ & 1 & 1.06 & $0.87,1.29$ & 0.82 & $0.65,1.02$ & 0.79 & $0.53,1.19$ & 1 & 0.72 & $0.44,1.16$ & 0.61 & $0.37,1.02$ & 0.51 & $0.19,1.33$ \\
\hline \multicolumn{15}{|l|}{ Death from CVD } \\
\hline No. of deaths & 147 & 82 & - & 63 & - & 13 & - & 14 & 27 & - & 18 & - & 4 & - \\
\hline Multivariate-adjusted ${ }^{*}$ & 1 & 0.87 & $0.65,1.18$ & 0.66 & $0.47,0.93$ & 0.76 & $0.42,1.39$ & 1 & 1.63 & $0.66,4.01$ & 1.49 & $0.58,3.80$ & 0.97 & $0.19,4.82$ \\
\hline \multicolumn{15}{|l|}{ Death from cancer } \\
\hline No. of deaths & 70 & 67 & - & 81 & - & 15 & - & 12 & 25 & - & 35 & - & 3 & - \\
\hline Multivariate-adjusted ${ }^{*}$ & 1 & 1.45 & $0.99,2.11$ & 1.37 & $0.93,2.02$ & 1.24 & $0.61,2.55$ & 1 & 0.87 & $0.39,1.92$ & 0.76 & $0.34,1.71$ & 0.62 & $0.13,2.93$ \\
\hline \multicolumn{15}{|l|}{ Non-smokers } \\
\hline \multicolumn{15}{|l|}{ Death from all causes } \\
\hline Person-years & 21962 & 15652 & - & 11120 & - & 833 & - & 30100 & 27158 & - & 16927 & - & 995 & - \\
\hline No. of deaths & 378 & 209 & - & 122 & - & 3 & - & 270 & 164 & - & 65 & - & 3 & - \\
\hline Multivariate-adjusted ${ }^{*}$ & 1 & 0.89 & $0.72,1.09$ & 0.89 & $0.68,1.14$ & 0.42 & $0.14,1.32$ & 1 & 0.76 & $0.59,0.97$ & 0.61 & $0.43,0.87$ & 0.47 & $0.12,1.92$ \\
\hline \multicolumn{15}{|l|}{ Death from CVD } \\
\hline No. of deaths & 187 & 91 & - & 49 & - & 1 & - & 134 & 58 & - & 22 & - & 0 & - \\
\hline Multivariate-adjusted* & 1 & 0.92 & $0.68,1.25$ & 0.79 & $0.52,1.21$ & 0.33 & $0.05,2.36$ & 1 & 0.58 & $0.38,0.87$ & 0.70 & $0.39,1.25$ & 0.00 & $0.00,0.00$ \\
\hline \multicolumn{15}{|l|}{ Death from cancer } \\
\hline No. of deaths & 113 & 82 & - & 51 & - & 0 & - & 92 & 70 & - & 32 & - & 3 & - \\
\hline Multivariate-adjusted ${ }^{*}$ & 1 & 0.98 & $0.69,1.39$ & $1 \cdot 11$ & $0.74,1.66$ & 0.00 & $0.00,0.00$ & 1 & 0.85 & $0.59,1.23$ & 0.59 & $0.35,0.99$ & 1.09 & $0.27,4.53$ \\
\hline
\end{tabular}

Ref., reference category.

*Adjusted for age (continuous), gender, smoking status (yes/no), BMI (continuous), educational level (low, medium, high), physical activity (low, medium, high), alcohol intake (>12 g/d), hypertension, diabetes, hypercholesterolaemia, history of CVD or cancer, family history of CVD, family history of cancer, total energy intake (continuous), vitamin supplement use, SFA, PUFA and $n-3$ PUFA intake (continuous), and menopausa status (in women). 
Table 4 Stratified analysis by alcohol intake of multivariate-adjusted hazard ratios (HR) and $95 \%$ confidence intervals for all-cause, CVD and cancer mortality by coffee consumption in men and women living in the Czech Republic, Poland, and Russia over a period of 6 years; the HAPIEE (Health, Alcohol and Psychosocial factors In Eastern Europe) study

\begin{tabular}{|c|c|c|c|c|c|c|c|c|c|c|c|c|c|c|}
\hline & \multicolumn{7}{|c|}{ Coffee consumption, men } & \multicolumn{7}{|c|}{ Coffee consumption, women } \\
\hline & \multirow{2}{*}{$\frac{<1 \text { cup/d }}{\text { Ref. }}$} & \multicolumn{2}{|c|}{$1-2$ cups/d } & \multicolumn{2}{|c|}{ 3-4 cups/d } & \multicolumn{2}{|c|}{$>4 \mathrm{cups} / \mathrm{d}$} & \multirow{2}{*}{$\frac{<1 \text { cup } / \mathrm{d}}{\text { Ref. }}$} & \multicolumn{2}{|c|}{$1-2$ cups/d } & \multicolumn{2}{|c|}{ 3-4 cups/d } & \multicolumn{2}{|c|}{$>4$ cups $/ \mathrm{d}$} \\
\hline & & $\mathrm{HR}$ & $95 \% \mathrm{Cl}$ & HR & $95 \% \mathrm{Cl}$ & $\mathrm{HR}$ & $95 \% \mathrm{Cl}$ & & $\mathrm{HR}$ & $95 \% \mathrm{Cl}$ & $\mathrm{HR}$ & $95 \% \mathrm{Cl}$ & $\mathrm{HR}$ & $95 \% \mathrm{Cl}$ \\
\hline \multicolumn{15}{|l|}{ Alcohol intake >12 g/d } \\
\hline \multicolumn{15}{|l|}{ Death from all causes } \\
\hline Person-years & 6357 & 4916 & - & 5293 & - & 602 & - & 404 & 771 & - & 1104 & - & 107 & - \\
\hline No. of deaths & 117 & 80 & - & 68 & - & 9 & - & 8 & 4 & - & 6 & - & 0 & - \\
\hline Multivariate-adjusted & 1 & 0.96 & $0.70,1.33$ & 0.70 & $0.49,0.99$ & 0.75 & $0.35,1.63$ & 1 & 0.22 & $0.04,1.17$ & $0 \cdot 10$ & $0.02,0.51$ & NA & NA \\
\hline \multicolumn{15}{|l|}{ Death from CVD } \\
\hline No. of deaths & 50 & 26 & - & 17 & - & 4 & - & 3 & 0 & - & 2 & - & 0 & - \\
\hline Multivariate-adjusted ${ }^{*}$ & 1 & 0.69 & $0.41,1.17$ & 0.35 & $0.18,0.69$ & 0.75 & $0.23,2.45$ & NA & NA & NA & NA & NA & NA & NA \\
\hline \multicolumn{15}{|l|}{ Death from cancer } \\
\hline No. of deaths & 28 & 29 & - & 35 & - & 5 & - & 2 & 2 & - & 4 & - & 0 & - \\
\hline Multivariate-adjusted* & 1 & 1.54 & $0.86,2.76$ & 1.66 & $0.93,2.97$ & 2.07 & $0 \cdot 70,6 \cdot 10$ & NA & NA & NA & NA & NA & NA & NA \\
\hline \multicolumn{15}{|l|}{ Alcohol intake $\leq 12 \mathrm{~g} / \mathrm{d}$} \\
\hline \multicolumn{15}{|l|}{ Death from all causes } \\
\hline Person-years & 25328 & 19199 & - & 15771 & - & 2253 & - & 32628 & 32797 & - & 24725 & - & 2060 & - \\
\hline No. of deaths & 556 & 340 & - & 235 & - & 27 & - & 311 & 226 & - & 119 & - & 11 & - \\
\hline Multivariate-adjusted* ${ }^{*}$ & 1 & 0.88 & $0.75,1.03$ & 0.74 & $0.62,0.89$ & 0.61 & $0.39,0.94$ & 1 & 0.73 & $0.59,0.90$ & 0.58 & $0.44,0.76$ & 0.50 & $0.23,1.08$ \\
\hline \multicolumn{15}{|l|}{ Death from CVD } \\
\hline No. of deaths & 285 & 147 & - & 95 & - & 10 & - & 145 & 86 & - & 39 & - & 4 & - \\
\hline Multivariate-adjusted ${ }^{*}$ & 1 & 0.78 & $0.67,0.98$ & 0.61 & $0.46,0.81$ & 0.57 & $0.30,1 \cdot 10$ & 1 & 0.56 & $0.40,0.80$ & 0.60 & $0.39,0.93$ & 0.38 & $0.09,1.60$ \\
\hline \multicolumn{15}{|l|}{ Death from cancer } \\
\hline No. of deaths & 156 & 122 & - & 97 & - & 10 & - & 102 & 93 & - & 64 & - & 6 & - \\
\hline Multivariate-adjusted* ${ }^{*}$ & 1 & 1.09 & $0.83,1.44$ & $1 \cdot 10$ & $0.81,1.49$ & 0.55 & $0.22,1.36$ & 1 & 0.94 & $0.68,1.30$ & 0.71 & $0.47,1.07$ & 0.76 & $0.27,2.12$ \\
\hline
\end{tabular}

Ref., reference category; NA, not applicable.

*Adjusted for age (continuous) gender, smoking status (ves/no), BMI (continuous), educational level (low, medium, high), physical activity (low, medium, high), smoking status (yes/no), hypertension, diabetes, hypercholesterolaemia, history of CVD or cancer, family history of CVD, family history of cancer, total energy intake (continuous), vitamin supplement use, SFA, PUFA and $n-3$ PUFA intake (continuous) and menopausal status (in women). 
other cohort studies specifically explored the association between coffee consumption and cancer mortality, reporting a decreased mortality risk from cancer ${ }^{(28)}$. Moreover, recent meta-analyses including epidemiological studies showed a protective effect of coffee consumption against liver, colorectal, breast and prostate cancer incidence ${ }^{(6)}$. Coffee has been hypothesized to play a role in the early phases of cancer development and promotion, delaying disease progression and thus prolonging a person's lifespan. As we found a significant association between coffee consumption and cancer mortality only in non-smoker women, we may hypothesize that smoking status might be a strong confounding factor and that the potentially beneficial effects of coffee are appreciable only when analysis is restricted to individuals free of the confounding effect of smoking. Another hypothesis is that the harmful effects of smoking among smokers counteract the potentially protective effect of coffee. However, some questions remain, for instance the lack of an evident decreased risk among non-smoker men. Knowledge of the type of cancer could help to draw more accurate hypotheses and explanations, but the data presented in the current study lack this information and future studies should take into account our results to better design their analyses.

The polyphenol content of coffee represents the main biological explanation for its benefits on health. During recent years, dietary patterns rich in polyphenols, such as the Mediterranean diet, have been suggested to exert their benefits due to their polyphenol content that may contribute towards their protective effects against diseases with an inflammatory basis ${ }^{(29)}$. Coffee has lately been demonstrated to be among the main contributors to polyphenol content in the diet of individuals living in Eastern European countries ${ }^{(30-34)}$ as well as among the foods with the highest antioxidant capacity ${ }^{(35)}$. Among the most important, chlorogenic acids are molecules which belong to the group of hydroxycinnamic acids demonstrating the ability to affect some metabolic pathways, for instance by improving glucose metabolism and decreasing inflammation and endothelial dysfunction ${ }^{(36)}$. This family of molecules has also demonstrated anti-tumour properties by inhibiting key enzymes involved in tumour genesis and metastasis $^{(37)}$. Other components, such as the diterpenes kahweol and cafestol, may play a role in the regulation of angiogenesis, apoptosis and inflammation processes and have been proposed as possible anti-tumour agents ${ }^{(38,39)}$. Together with these effects, an excess of the same compounds, for instance caffeine and diterpenes, may produce harmful effects, such as an acute rise in blood pressure, increase in serum cholesterol and homocysteine levels, and decreased insulin sensitivity, all contributing to raise the risk for $\mathrm{CVD}^{(23,40,41)}$. The equilibrium between positive and negative effects on health may explain the findings of our and other studies, as an excessive consumption was not associated with the same benefits as moderate daily intake. The antioxidant compounds are thought to counteract the potentially harmful effects of caffeine or diterpenes, but an excessive intake of the latter may disrupt such balance, thus weakening or even annulling the overall beneficial effect of coffee. Moreover, other factors may impact the effects of coffee consumption on health. For instance, some previous studies have emphasized the possible role of associated lifestyle habits, such as smoking status, which largely correlates with mortality. Also in the present study we confirmed that current smoking, associated with coffee drinking, was weakening the effect of coffee on CVD mortality, as a stronger association between coffee and CVD mortality was found among non-smokers. In light also of the aforementioned findings obtained on cancer mortality, an accurate study of smoking status and coffee drinking should be conducted in future investigations aimed to explain the effects of coffee on human health, whatever is the outcome.

It is still debatable if the health status of a person may influence coffee consumption (i.e. those with poorer health conditions drinking less coffee), thus biasing the results. Generally, studies on coffee consumption and mortality report analyses with the exclusion of deaths occurring within the first 2 years from baseline observation with no substantial changing of the results. In our study we performed a similar analysis and the results suggested that health status at baseline did not necessarily explain the aforementioned association. In addition, we also took into account health status by adjusting for a previous history of CVD and cancer, as well as the presence of CVD risk factors, in the final model of multivariable analysis and excluded these cases in an additional sub-analysis in order to reveal possible reverse causation. Besides these strengths, the current study also presented some limitations. First, the data presented refer only to the baseline examination and coffee consumption was self-reported. Therefore, we were not able to control for possible variation of coffee intake during the follow-up period. Second, data regarding the type of coffee and methods of preparation were not collected. Thus, we can only assume homogeneous preparation methods within and between countries, but differences regarding the use of caffeinated and decaffeinated coffee may still exist. Third, the study had relatively short follow-up, thus cases are limited, especially regarding cancer mortality. Fourth, the last category of exposure included fewer individuals than others and this could have affected the statistical power. Nevertheless, for analysis, we preferred to consider this category as separate due to a different biological effect of extreme coffee consumption. Finally, the sample was recruited in urban centres and thus should not be considered nationally representative.

\section{Conclusion}

In conclusion, in our cohort coffee consumption was associated with decreased risk of all-cause and CVD 
mortality. The healthful effect of coffee seems to rely on CVD, but additional analyses focused on non-smokers are needed to further investigate potentially preventive effects also against cancer.

\section{Acknowledgements}

Financial support: The study was funded by the Wellcome Trust (grant numbers 064947/Z/01/Z and 081081/Z/06/Z); the US National Institute on Aging (grant number 1R01 AG23522-01); and the MacArthur Foundation Initiative on Social Upheaval and Health (award number 71208). The funders had no role in the design, analysis or writing of this article. Conflict of interest: The authors declare that they have no conflict of interest. Authorship: G.G. designed the study, performed the analysis and drafted the manuscript. A.M. performed the analysis. U.S. contributed in collecting data. D.S. provided critical revision for the data analysis methods. A.P. and M.B. designed the study and provided critical revision. All authors provided final approval for the manuscript. Ethics of human subject participation: This study was conducted according to the guidelines laid down in the Declaration of Helsinki and all procedures involving human subjects were approved by the University College London Hospital research ethics committee and by ethical committees in each participating country. Written informed consent was obtained from all subjects.

\section{Supplementary material}

To view supplementary material for this article, please visit http://dx.doi.org/10.1017/S1368980016001749

\section{References}

1. Grigg D (2003) The worlds of tea and coffee: patterns of consumption. GeoJournal 57, 283-294.

2. Cano-Marquina A, Tarin JJ \& Cano A (2013) The impact of coffee on health. Maturitas 75, 7-21.

3. Jiang X, Zhang D \& Jiang W (2014) Coffee and caffeine intake and incidence of type 2 diabetes mellitus: a meta-analysis of prospective studies. Eur J Nutr 53, 25-38.

4. Steffen M, Kuhle C, Hensrud D et al. (2012) The effect of coffee consumption on blood pressure and the development of hypertension: a systematic review and meta-analysis. J Hypertens 30, 2245-2254.

5. Ding M, Bhupathiraju SN, Satija A et al. (2014) Long-term coffee consumption and risk of cardiovascular disease: a systematic review and a dose-response meta-analysis of prospective cohort studies. Circulation 129, 643-659.

6. Bohn SK, Blomhoff R \& Paur I (2014) Coffee and cancer risk, epidemiological evidence, and molecular mechanisms. Mol Nutr Food Res 58, 915-930.

7. Corti R, Binggeli C, Sudano I et al. (2002) Coffee acutely increases sympathetic nerve activity and blood pressure independently of caffeine content: role of habitual versus nonhabitual drinking. Circulation 106, 2935-2940.

8. Godos J, Pluchinotta FR, Marventano S et al. (2014) Coffee components and cardiovascular risk: beneficial and detrimental effects. Int J Food Sci Nutr 65, 925-936.
9. Ludwig IA, Clifford MN, Lean ME et al. (2014) Coffee: biochemistry and potential impact on health. Food Funct $\mathbf{5}$, $1695-1717$.

10. Ahmed HN, Levitan EB, Wolk A et al. (2009) Coffee consumption and risk of heart failure in men: an analysis from the Cohort of Swedish Men. Am Heart J 158, 667-672.

11. Happonen P, Laara E, Hiltunen L et al. (2008) Coffee consumption and mortality in a 14-year follow-up of an elderly northern Finnish population. Br J Nutr 99, 1354-1361.

12. Laaksonen $\mathrm{M}$, Talala $\mathrm{K}$, Martelin $\mathrm{T}$ et al. (2008) Health behaviours as explanations for educational level differences in cardiovascular and all-cause mortality: a follow-up of 60000 men and women over 23 years. Eur J Public Health 18, 38-43.

13. Leurs LJ, Schouten LJ, Goldbohm RA et al. (2010) Total fluid and specific beverage intake and mortality due to IHD and stroke in the Netherlands Cohort Study. Br J Nutr 104, 1212-1221.

14. Grosso G, Stepaniak U, Micek A et al. (2014) Association of daily coffee and tea consumption and metabolic syndrome: results from the Polish arm of the HAPIEE study. Eur J Nutr 54, 1129-1137.

15. Grosso G, Stepaniak U, Polak M et al. (2015) Coffee consumption and risk of hypertension in the Polish arm of the HAPIEE cohort study. Eur J Clin Nutr 70, 109-115.

16. Peasey A, Bobak M, Kubinova R et al. (2006) Determinants of cardiovascular disease and other non-communicable diseases in Central and Eastern Europe: rationale and design of the HAPIEE study. BMC Public Health 6, 255.

17. Willett WC, Sampson L, Stampfer MJ et al. (1985) Reproducibility and validity of a semiquantitative food frequency questionnaire. Am J Epidemiol 122, 51-65.

18. Brunner E, Stallone D, Juneja M et al. (2001) Dietary assessment in Whitehall II: comparison of $7 \mathrm{~d}$ diet diary and food-frequency questionnaire and validity against biomarkers. Br J Nutr 86, 405-414.

19. Lof M, Sandin S, Yin L et al. (2015) Prospective study of coffee consumption and all-cause, cancer, and cardiovascular mortality in Swedish women. Eur J Epidemiol 30, $1027-1034$.

20. Crippa A, Discacciati A, Larsson SC et al. (2014) Coffee consumption and mortality from all causes, cardiovascular disease, and cancer: a dose-response meta-analysis. $A m \mathrm{~J}$ Epidemiol 180, 763-775.

21. Greenberg JA, Dunbar CC, Schnoll R et al. (2007) Caffeinated beverage intake and the risk of heart disease mortality in the elderly: a prospective analysis. Am J Clin Nutr 85, 392-398.

22. Rosner SA, Akesson A, Stampfer MJ et al. (2007) Coffee consumption and risk of myocardial infarction among older Swedish women. Am J Epidemiol 165, 288-293.

23. Verhoef P, Pasman WJ, Van Vliet T et al. (2002) Contribution of caffeine to the homocysteine-raising effect of coffee: a randomized controlled trial in humans. Am J Clin Nutr $\mathbf{7 6}$, 1244-1248.

24. Ammon HP, Bieck PR, Mandalaz D et al. (1983) Adaptation of blood pressure to continuous heavy coffee drinking in young volunteers. A double-blind crossover study. Br J Clin Pharmacol 15, 701-706.

25. Grosso G, Marventano S, Galvano F et al. (2014) Factors associated with metabolic syndrome in a mediterranean population: role of caffeinated beverages. J Epidemiol 24, 327-333.

26. Myers MG (1988) Effects of caffeine on blood pressure. Arch Intern Med 148, 1189-1193.

27. Robertson D, Wade D, Workman R et al. (1981) Tolerance to the humoral and hemodynamic effects of caffeine in man. J Clin Invest 67, 1111-1117. 
28. Tamakoshi A, Lin Y, Kawado M et al. (2011) Effect of coffee consumption on all-cause and total cancer mortality: findings from the JACC study. Eur J Epidemiol 26, 285-293.

29. Grosso G, Marventano S, Yang J et al. (2015) A comprehensive meta-analysis on evidence of Mediterranean diet and cardiovascular disease: are individual components equal? Crit Rev Food Sci Nutr (Epublication ahead of print version).

30. Grosso G, Stepaniak U, Topor-Madry R et al. (2014) Estimated dietary intake and major food sources of polyphenols in the Polish arm of the HAPIEE study. Nutrition 30, 1398-1403.

31. Perez-Jimenez J, Fezeu L, Touvier M et al. (2011) Dietary intake of 337 polyphenols in French adults. Am J Clin Nutr 93, 1220-1228.

32. Tresserra-Rimbau A, Medina-Remon A, Perez-Jimenez J et al. (2013) Dietary intake and major food sources of polyphenols in a Spanish population at high cardiovascular risk: the PREDIMED study. Nutr Metab Cardiovasc Dis 23 , 953-959.

33. Zamora-Ros R, Knaze V, Rothwell JA et al. (2015) Dietary polyphenol intake in Europe: the European Prospective Investigation into Cancer and Nutrition (EPIC) study. Eur J Nutr 55, 1359-1375.

34. Zujko ME, Witkowska AM, Waskiewicz A et al. (2012) Estimation of dietary intake and patterns of polyphenol consumption in Polish adult population. Adv Med Sci 57, 375-384.
35. Pellegrini N, Serafini M, Colombi B et al. (2003) Total antioxidant capacity of plant foods, beverages and oils consumed in Italy assessed by three different in vitro assays. J Nutr 133, 2812-2819.

36. Lopez-Garcia E, van Dam RM, Qi L et al. (2006) Coffee consumption and markers of inflammation and endothelial dysfunction in healthy and diabetic women. Am J Clin Nutr 84, 888-893.

37. Jin UH, Lee JY, Kang SK et al. (2005) A phenolic compound, 5 -caffeoylquinic acid (chlorogenic acid), is a new type and strong matrix metalloproteinase-9 inhibitor: isolation and identification from methanol extract of Euonymus alatus. Life Sci 77, 2760-2769.

38. Cardenas C, Quesada AR \& Medina MA (2011) Anti-angiogenic and anti-inflammatory properties of kahweol, a coffee diterpene. PLoS One 6, e23407.

39. Wang S, Yoon YC, Sung MJ et al. (2012) Antiangiogenic properties of cafestol, a coffee diterpene, in human umbilical vein endothelial cells. Biochem Biophys Res commun 421, 567-571.

40. Hartley TR, Lovallo WR \& Whitsett TL (2004) Cardiovascular effects of caffeine in men and women. Am J Cardiol 93, 1022-1026.

41. Thong FS \& Graham TE (2002) Caffeine-induced impairment of glucose tolerance is abolished by $\beta$-adrenergic receptor blockade in humans. I Appl Physiol 92, 2347-2352. 